\title{
Gender difference in learning disabled children Neuropsychological review
}

\author{
Mohamad Qasim Abdullah* \\ Department of Counselling Psychology, University of Aleppo, Syria
}

Received: 些 March 05, 2018; Published: 眥 March 09, 2018

*Corresponding author: Mohamad Qasim Abdullah, Department of Counselling Psychology, Faculty of Education, University of Aleppo, Syria

\begin{abstract}
This review aimed to investigate the gender differences in learning disabilities among children. It is highlighted the nature of learning disabilities, and reviewed the gender differences on one hand, and explanation of theoretical background on the other hand. Finally, it is centred on the neuropsychology of these complicated behavioral phenomena. The conclusion of this article is that we are in the midst of investigating differences between male and female from multidimensional and methodological approaches because there are some critical issues regarding the assessing this complicated behavioural phenomena.
\end{abstract}

Keywords: Learning disabilities; Gender differences; Children

\section{Introduction}

Learning disabilities (LD) are one of the most significant causes of school failure and may result from different causes and cognitive processes. "LD" refers to a variety of disorders that affect the acquisition, retention, understanding, organization or use of verbal and/ or non-verbal information. According to the definition, learning disabilities are due to genetic, congenital and acquired neurobiological factors that result in impairments in one or more psychological processes related to learning. In view of the neurobio psychological, nature of the disability, the formulation and communication of a diagnosis of learning disabilities is complex process that requires professional training and skill for assessing, and knowing the gender differences of neuropsychological structure of the brain [1]. LDs are specific not global impairments and as such are distinct from intellectual disabilities.

Learning disorders are among the most frequently diagnosed developmental disorders in childhood. Epidemiological studies report comparable prevalence rates of $4-9 \%$ for deficits in reading and $3-7 \%$ for deficits in mathematics (DSM-5). More recently, studies have started examining the relationship between deficits in different learning domains (i.e., deficits in reading and deficits in mathematics) in order to better understand their overlap, rather than focusing on a single deficit only. Findings suggest that children experiencing a deficit in one learning domain frequently show deficits in other domains as well (Badian N 1983). Furthermore, be haviour-genetic analyses provide evidence that reading and mathematics disorders share genetic variance [2].

The World Health Organization has, in the past, used a three-tier hierarchy of terms to describe the variations in human function, as related to disability issues. These were impairment, disability and handicap.

i. Impairment was defined as "loss or abnormality of psychological, physiological or anatomical structure or function". This made impairment "intrinsic to the individual" (a phrase that appears in a number of other definitions of learning disabilities). Impairment is either functional or medical in nature and a learning disability is usually considered neurological impairment.

ii. Impairment became a disability, when the individual with impairment was expected to carry out regular or routine tasks that relied on the use of skills or knowledge in the areas, affected by the impairment.

iii. The third level in this hierarchy was the term "handicap", which described the impact of the disability, when there were no accommodations or supports offered to the individual concerned. Most jurisdictions have eliminated the term "handicap" from legislation and regular use $[3,4]$. 
LDs range in severity and invariably interfere with the acquisition and use of one or more of the following important skills:

a. Oral language (e.g., listening, speaking, understanding)

b. Reading (e.g., decoding, comprehension)

c. written language (e.g., spelling, written expression)

d. Mathematics (e.g., computation, problem solving)

Eliminated from usage until such time that the Human Rights Code is amended. People with LD usually have difficulties acquiring, retaining, processing, understanding, organizing and using information in all of its diverse forms, and included among "communication exceptionalities". In lay terms, they are often described as problems with information processing, especially verbal and non-verbal, symbolic and concrete information [5]. LDs may also cause difficulties with organizational skills, social perception and social interaction. The impairments are generally life-long. For example, Reading disabilities (RD) are common and may have a lasting effect on children's academic life, self-esteem, and professional achievement [6].

However, their effects may be expressed differently over time, depending on the match between the demands of the environment and the individual's characteristics. Some impairment may be noted during the pre-school years, while others may not become evident until much later. During the school years, learning disabilities are suggested by unexpectedly, low academic achievement or achievement that is sustainable only by extremely high levels of effort and support [7].

\section{Gender differences in learning disabilities:}

At first sight, LDs appear to be more common among schoolaged boys than girls are. About two-thirds of school-age students identified with learning disabilities are males. The research on learning disabilities (LD) purported that the ratio of boys to girls with learning disabilities was between 5:1 and 9:1, respectively, in the school-identified population. . In addition, not by just a little bit. Boys make up around two-thirds of kids identified as having what IDEA refers to as a "specific learning disability." In addition, boys are also more than twice as likely to be identified as having ADHD. But saying that more boys are identified as having learning and attention issues doesn't mean that boys actually have learning and attention issues more often than girls do. However, a recent, comprehensive study conducted has demonstrated an equal number of boys and girls having learning disabilities. It seems that way because, in fact, more boys than girls are formally identified as having learning disabilities and ADHD. LD and ADHD are the two most commonly diagnosed learning and behavioural problems in schools [8].

Boys more often the girls are affected by all the cognitive developmental disorders of childhood. Differences in the etiology of learning disabilities, as well as general differences in learning styles in boys versus girls may explain the male preponderance in the prevalence of learning disabilities [9]. A well-known study of 400 students found no gender gap when it came to learning issues. But that was using scientific criteria. When teachers made the call, they recommended twice as many boys for LD program. One reason may be the differences in behaviours between boys and girls. We know that boys in general tend to draw attention that is more negative in schools. One report showed that boys represent around 85 percent of all discipline referrals. Another showed that 22 percent of boys had been formally disciplined, versus 8 percent of girls $[10,11]$.

Additionally, it has be known that boys with ADHD tend to show more hyperactivity, impulsivity and physical aggression than girls with ADHD. That behaviour makes them stand out from the other boys. Boys with ADHD are more noticeable than girls with ADHD. In addition, that may explain why more than twice as many of them are identified with the disorder. We don't know whether boys actually have learning and attention issues more often than girls do. However, we do know that when kids are identified they are more likely to get the best support. If girls are under-identified, it means that many are not getting the help they need. So parents of girls who are struggling might have to be even stronger advocates for their child $[4,12]$.On the other hand, it is very significant to mention that, the temperament play an important role in child personality. With regard to motor activity level, boys on average show somewhat higher levels than girls do.

This differences increases with age until the early school years. At the high extreme of the scale the proportion of boys is at least twice the proportion of girls. Hyperactive motor behaviour is an important component of behavioural disorders, especially, hyperactivity and addition deficit disorder and distractibility. With respect to a causal explanation for this gender differences, some believe in worldwide role stereotyping, or worldwide constitutional factors. Kohnstamm [13] believed that the cross-cultural universality and the regularity of the differences found make a biological rather than a cultural explanation the most plausible [11,14]. Recent studies show that mathematics disorder is a learning disorder. Children with this disorder have math skills is much lower than mean for their age, intelligence, and education. The disorder affects the Child's success at school. It is thought that up to $7 \%$ of children have this disorder. It affects boys and girls equally. It is also caused dyscalculia.

The cause of this disorder is not known. Like other learning disorders, it occurs more in some families. Mathematics disorder may also be the result of damage in certain parts of the brain. It also has led to a weak understanding of mathematical concepts and increased realization of mathematics. Findings indicated that there is not meaningful difference between genders. Since this research was case study, it seems that this difference will be indicated in vast studies. Then it suggests that have to do more study in this field for its causes [1]. 


\section{Explanation of gender differences}

i. Biological Vulnerability: Many theories have been proposed to explain why more boys than girls are identified as having learning disabilities. Some researchers proposed that the increased prevalence is due to a child's biological vulnerability. This means that they may be born with or acquire a tendency for a learning disability early in life.

ii. Referral Matter: Other studies suggest that this discrepancy in identification may be due to referral bias. Boys are more likely to be referred for special education when they demonstrate academic problems because of other apparent behaviours. Boys who are frustrated and struggle academically are more likely to act out. They may be hyperactive, impulsive, or disruptive in class, while girls typically show less obvious signs of their academic frustrations. For instance, girls who only show inattention are more likely to be missed by teachers and seen as uninterested in the subject matter. This same ratio of boys to girls (5:1) is reported for ADHD as well.

iii. Test Matter: The true frequency of LDs among genders is subject to much dispute for many reasons. Some researchers say that the lack of a universal definition of "learning disability" and the absence of accurate, objective testing criteria to measure learning disabilities directly correlate to inaccurate identification of children with learning disabilities $[14,15]$. It should be noted that many of the tests used to diagnose LDs were designed and standardized on boys. Consequently, these tests might not address differences in the way that boys reveal their learning disabilities, as compared with girls. The tests may not address certain types of problems found specifically with girls $[1,3]$. Since the category of LD first arose in 1975, the number of students identified with learning disabilities has tripled. Approximately 2.4 million students are identified as having LD and receive special education services in schools [11,14].

iv. A number of reasons have been suggested for the vast increase in children diagnosed with learning disabilities. These reasons include:

a. Biological and psychosocial stressors may put more children at risk for having learning disabilities, and as a result, more children are identified,

b. The diagnosis of LD is more socially acceptable than many other special education classifications. There is reluctance on the part of teachers to label a child "mentally retarded" or "emotionally disturbed." Parents even prefer the "LD classification" and push for it.

c. Children who are academically underachieving are incorrectly labeled as individuals with learning disabilities [16]. The evaluation and diagnostic criteria may be too subjective, unre- liable, and flawed by nature. Furthermore, there may be few, if any, alternative programs for these underachieving students.

d. Greater overall awareness of learning disabilities and comprehensive analysis of student performances have resulted in more substantiated referrals and identifications. Teachers and parents are cognizant of the different types of services that are available to the students, $[5,13]$.

\section{Neuropsychology and Gender differences}

Before discussing the neuropsychology of learning disabilities, it is significant to describe the gender differences in learning. Two questions should be stated:

i. First: What did we learn?

a. Their differences in teaching methods, Females are more interpersonally oriented.

b. Males are more tasks oriented.

c. Males are more focused on teaching the material rather than how you are feeling or processing the material

d. If the task is completed; male instructors have done their job [17].

ii. Second: Are girls and boys that different? It should be noted, that:

a. We have all heard phrases similar to, "Men are from Mars, and Women are from Venus", but how different are they really?

b. In this presentation we will look at the different learning styles of boys and girls in school, as well as how teachers can cater to their varying needs

c. According to the Reader's Digest, boys and girls literally see the world around them differently.

d. This is due to differences in the physical composition of their eyes, ears, and brain. The male eye is drawn to cooler colors like silver, blue, black, grey, and brown

e. the composition of the male eye also makes it more attuned to motion and direction,

f. "Boys interpret the world as objects moving through space." Girls however, have eyes that are drawn to warmer colors (reds, oranges, yellow), as well as textures or visuals with more detail (i.e. faces).

g. Girls do not tend to see the world as objects in motion, thus it does not typically affect their learning $[3,7]$.

iii. Third: Differences in Brain Development: Another difference between boys and girls is how their brains develop. According to Gurian M [15], the actual brain structure differences have a 
substantial affect on learning, and to explore the dominance of different school subjects in boys and girls. The results concluded that brain structure was a direct causal factor. The researchers removed all gender bias, by not looking at whether or not the brains being observed were male or female [18].

It has been showed that there was a direct correlation in brain structure and the subjects preferred by the children. Generally speaking, the results showed that girls do not prefer physics as much as the boys did, but enjoyed reading and writing. Boys, on the other hand, took more to physics and mathematics, while disliking reading and writing. This is because boy's brains are spatial. This means that they are more prone to "use more abstract strategies such as derived facts or invented algorithms"that"reflected conceptual understanding." (Fennema, et al. [15]). Females are also less likely to have learning disabilities. This is because the female brain "uses more cortical areas for more learning functions". Thus, male and female brains are physiologically different, and these differences affect most of the learning processes $[1,3]$.

Researchers suggest that dyslexia may have different neural origins in each sex. MRI scans revealed that among males, and consistent with previous studies, those with dyslexia has less gray matter volume in brain areas such as the left temporal gyrus, which is involved in language. Among females, those with dyslexia had less grey matter volume in the right parietal lobe which is associated with sensory and motor processing. Surprisingly, they found no differences in the temporal lobes of females with dyslexia $[8,19] \mathrm{Neu}$ ropsychology studies the relationship between the human brain and behaviour. Its development as specific area of study is relatively recent, although its scientific basis results from several decades of knowledge and investigation. According to Cunha, neuropsychological screening initially sought to identify and locate focal brain lesions. Today, it is based upon the dynamic location of functions, with the aim of investigating higher cortical functions, such as attention, memory, language, among other [13].

The participation of the brain is regarded as a whole, where areas are interdependent and correlated, working like an orchestra, which needs the integration of its components to perform a concert. This is called Functional system. The major focus of this system is the development of human behaviour science based on brain functioning, [20]. This way, by knowing the normal development and functioning of the brain, we may understand cerebral abnormalities, such as cognitive and behavioural disorders that result from injuries, diseases or abnormal brain development. Child neuropsychology, whose aim is the early identification of cognitive and behavioural disorders, has become one of the key elements of regular well-child visits. In this case, appropriate tools (neuropsychological tests and developmental assessment scales) are necessary [5].The results of these scales and tests show the major gains during development and aim at determining the child's specific level of development.
The importance of these tools lies in the prevention and early detection of developmental and learning disabilities, carefully indicating the pace and quality of the process, allowing for qualitative and quantitative mapping. Of brain areas and their interconnections (functional system), with the aim of early and accurate therapeutic interventions [21]. In sum, the gender differences in learning disabled children is very complicated- behavioural phenomena, because it is related to childhood personality-temperament, in addition, to its psycho biosocial factors on one hand, and to the assessing instruments implemented by clinicians / researchers and participants, on the other hand. Given the complex nature of education, in addition to the intricacies of group and individual differences in academic achievement, more research is needed to illuminate the gender differences in LDs according to the development of cognitive processes and brain function. We look forward to investigate gender differences from multidimensional perspective for good assessing, understanding and intervention [22,23].

\section{References}

1. Haddad D (2017) Gender differences in learning disabilities. Learning disabilities Basics. HONcode standard for trustworthy health, USA.

2. Kovas, Y Haworth, C Harlaar, N Petrill S, Dale P, et al. (2007) Overlap and specificity of genetic and environmental influences on mathematics and reading disabilities in 10-year-old twins. Journal of Child Psychology and Psychiatry 48(9): 914-922.

3. Galaburda A (2011) Neuroscience, Education, and Learning Disabilities. Human Neuroplasticity and Education Pontifical Academy of Sciences, Scripta Varia 117, Vatican City, Europe.

4. Mayes S, Calhoun S, Crowwell E (2000) Learning disabilities and ADHD; overlapping spectrum disorders. Learning Disabilities 33 (5): 417-424.

5. NCLD (2014) The state of learning disabilities. National Center for Learning Disabilities. Park Avenue South, Suite, New York, USA.

6. Oakhill JV, Petrides A (2007) Sex differences in the effects of interest on boys' and girls' reading comprehension. British journal of Psychology 98: 223-235.

7. LDAO (2001) Learning disabilities: a new definition. Learning Disabilities Association of ONTAR I O. USA.

8. Lack C (2015) Gender differences in specific learning disorders.

9. Ruth D Nass M (1993) Sex differences in learning abilities and disabilities, Annals of Dyslexia 43: 61-77.

10. Berninger VW (2004) The reading brain in children and youth: A systems approach. In BYL Wong (Ed.) Learning about learning disabilities. San Diego, CA: Elsevier Academic Press pp. 197-248.

11. Vogel S, Walsh P (1987) Gender differences in cognitive abilities of leaning disabled females and males. Annals of Dyslexia 37(1): 142-156.

12. Karimi S (2013) is there gender differences between learning disabled students performance in mathematical activities 00030: 7.

13. Kohnstamm G (1989) Temperament in childhood: Cross-cultural and sex differences In: Geldolph Kohnstamm0020John Bates: Temperament in childhood. John Wiley \& Sons, New York, USA.

14. Jamie M, Quinn M, Wagner R (2013) Gender Differences in Reading impairment and in the Identification of Impaired Readers: Results From a Large-Scale Study of At-Risk Readers. Journal of learning XX(X): 1-13. 
15. Gurian M (2010) Boys and girls learn differently. Jossey-Bass, A Wiley imprint, Europe.

16. Hawke J L, Wadsworth S J, Olson R K, Defries J C (2007) Etiology of reading difficulties as a function of gender and severity. Reading and Writing 20: $13-25$.

17. Plomin R (2003) A genetic theory of learning disabilities.

18. Willcutt EG, Petrill SA, Wu S, Boada R, DeFries JC, et al. (2013) Comorbidity Between Reading Disability and Math Disability Concurrent Psychopathology, Functional Impairment, and Neuropsychological Functioning. Journal of learning disabilities 46: 500-516.

19. Halpern D (2004) A cognitive-process taxonomy for sex differences in cognitive abilities. Current Directions in Psychological Science 13(4): 135-139.

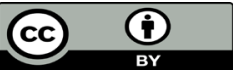

This work is licensed under Creative Commons Attribution 4.0 License

To Submit Your Article Click Here : Submit Article

DOI: 10.32474/RRHOAJ.2018.01.000111
20. Landerl K, Göbel M, Moll K (2013) Core deficit and individual manifestations of developmental dyscalculia (DD): the role of comorbidity. Trends in Neuroscience and Education 2: 38-42.

21. Danielle L, Azambuja L, Mirna P, Jaderson C (2004) Neuropsychological assessment in children. Jornal de Pediatria.

22. American Psychiatric Association (2013) DSM 5: American Psychiatric Association, USA.

23. Kaufman A (2017) Neuropsychology and specific learning disabilities: Lessons from the past as a guide to present controversies and future clinical practice. Neuropsychological Perspectives on Learning Disabilities in the Era of RTI, USA.

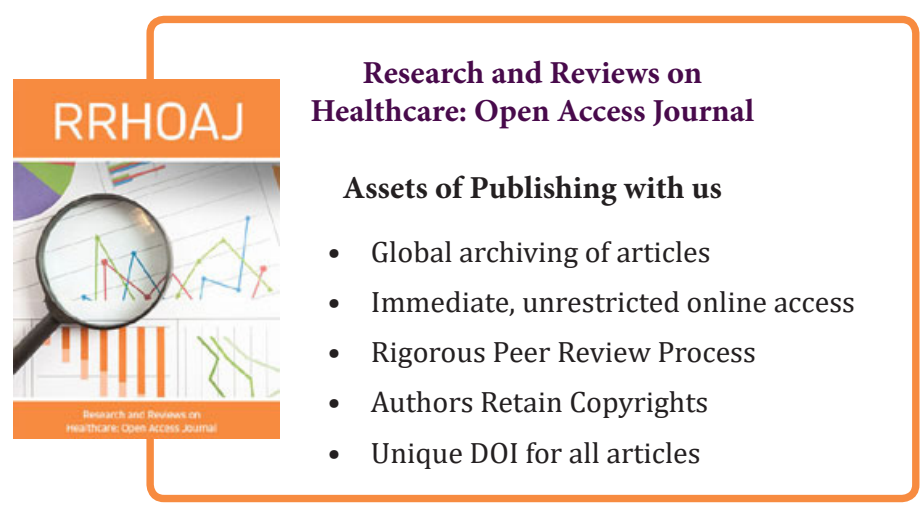

